\title{
Long-term outcomes of trials in the National Institute for Health and Care Excellence depression guideline
}

\author{
Susan McPherson and Michael P. Hengartner
}

\section{Summary}

The forthcoming National Institute for Health and Care Excellence depression guideline reviews short-term outcomes for long-term depression. We present effect sizes for long-term outcomes in trials that report these data. Psychological therapies become more effective, whereas antidepressants become less effective over the long term. We review other forms of longitudinal research that support these findings.

Declaration of interest

None.

\section{Keywords}

Antidepressants; depressive disorders; individual psychotherapy; outcome studies.

\section{Copyright and usage}

(c) The Author(s) 2019. This is an Open Access article, distributed under the terms of the Creative Commons Attribution licence (http://creativecommons.org/licenses/by/4.0/), which permits unrestricted re-use, distribution, and reproduction in any medium, provided the original work is properly cited.
The National Institute for Health and Care Excellence (NICE) have compiled and synthesised a large collection of randomised controlled trials on depression for the forthcoming guideline update. ${ }^{1}$ Trials on long-term forms of depression are grouped into chronic depression and treatment-resistant depression (TRD). Chronic depression trial populations (defined as an episode of at least 2 years) have an actual range of 26-348 months duration. ${ }^{1}$ TRD trial populations have episodes ranging from 0.4 to 92 months; $60 \%$ of TRD studies report mean episodes of 2 years or more. ${ }^{1}$ Long-term outcomes in these trials have not been analysed; the review only uses end-of-treatment outcomes, which vary from 1 to 78 weeks, with an average of around 10 weeks. The rationale given is that there are not enough studies with long-term data to analyse. ${ }^{2}$ Stakeholders have argued that long-term outcomes provide the 'best-possible evidence' and NICE executives have recently agreed to look at the issue again. ${ }^{3} \mathrm{We}$ examine available data to illustrate how NICE could make use of this evidence. Although NICE and other commentators consider TRD and chronic depression to be distinct categories of depression, it has been argued that when subclassified based on limited information available from trials reviewed by NICE, these are not clinically meaningful distinctions. ${ }^{4}$ Specifically, based on the information both present and, critically, absent in the trial papers, there is no reliable basis on which to suppose that these populations are not overlapping. We therefore examine both categories for the purposes of the current commentary.

In chronic depression and TRD categories, 124 trials were included. ${ }^{1}$ Ninety-eight of these included an antidepressant arm (as defined by NICE), 30 included a psychological treatment arm and a proportion of these had both antidepressant and psychological treatment groups. Twenty-two had observation points of 6 months or more. Of these, there were 11 in which the 6-month observation was at end of treatment, rather than 'follow-up'. Without a period between end of treatment and follow-up, it is not possible to form a view on whether the effects of the treatment last beyond end of treatment. Table 1 collates information for the remaining 11 trials: we report the amount of change on continuous symptom scales at end of treatment and follow-up for each of the trials with long-term follow-up data via Cohen's $d$ effect sizes. Trials are ordered by group comparison effect size at follow-up.
Time between end of treatment and follow-up ranged from 18 to 104 weeks, which limits comparability. In addition, in the smaller studies, the confidence intervals were quite wide, which limits the degree of certainty in the findings. However, examining pre-post effect sizes by intervention arm indicates that most psychological treatments for both TRD and chronic depression appear to become more effective at follow-up, with the exception of cognitive interpersonal group psychotherapy, which appears to become less effective, and cognitive-behavioural analysis system of psychotherapy, which has mixed results. The latter two psychological treatments were examined in populations classified by NICE as chronic depression. Examining group comparison effect sizes at follow-up suggests that antidepressants are consistently less effective than either psychological treatment alone or in combination with antidepressants over the long term.

There are limitations to comparing antidepressant and psychological treatment trials, which apply to all the trials reviewed, including inflation of outcomes from different comparator arms (only two of the trials directly compared antidepressants alone with psychological treatment alone); effects of completer versus intention-totreat (ITT) analyses (half of the trials here used ITT or modified ITT requiring a minimum of one session attendance); inflation of effects from non-blinding in psychological treatments; and differential effects of under-reporting harms, side-effects and tolerability in psychological treatment versus antidepressant trials. Therefore, the findings here are not presented as a standalone meta-analysis and should be interpreted with caution. NICE guidelines provide grading of recommendations assessment, development and evaluation (GRADE) of all trials to take these sorts of bias into account when translating findings into recommendations. Although the draft depression guideline has produced preliminary GRADE assessments, these cannot yet be directly applied to the current analysis because they remain under review and stakeholders have raised significant concerns about the way in which GRADE was applied. ${ }^{2}$ Of particular relevance to the current analysis is the concern that trials were downrated on quality if the $95 \%$ confidence interval crossed both line of no effect and threshold for clinically important benefit at the end of treatment, irrespective of whether this had ceased to be the case at a follow-up data point, which stakeholders argue prejudices trials where the effect emerges at follow-up. Also relevant is the concern that trials were downrated 


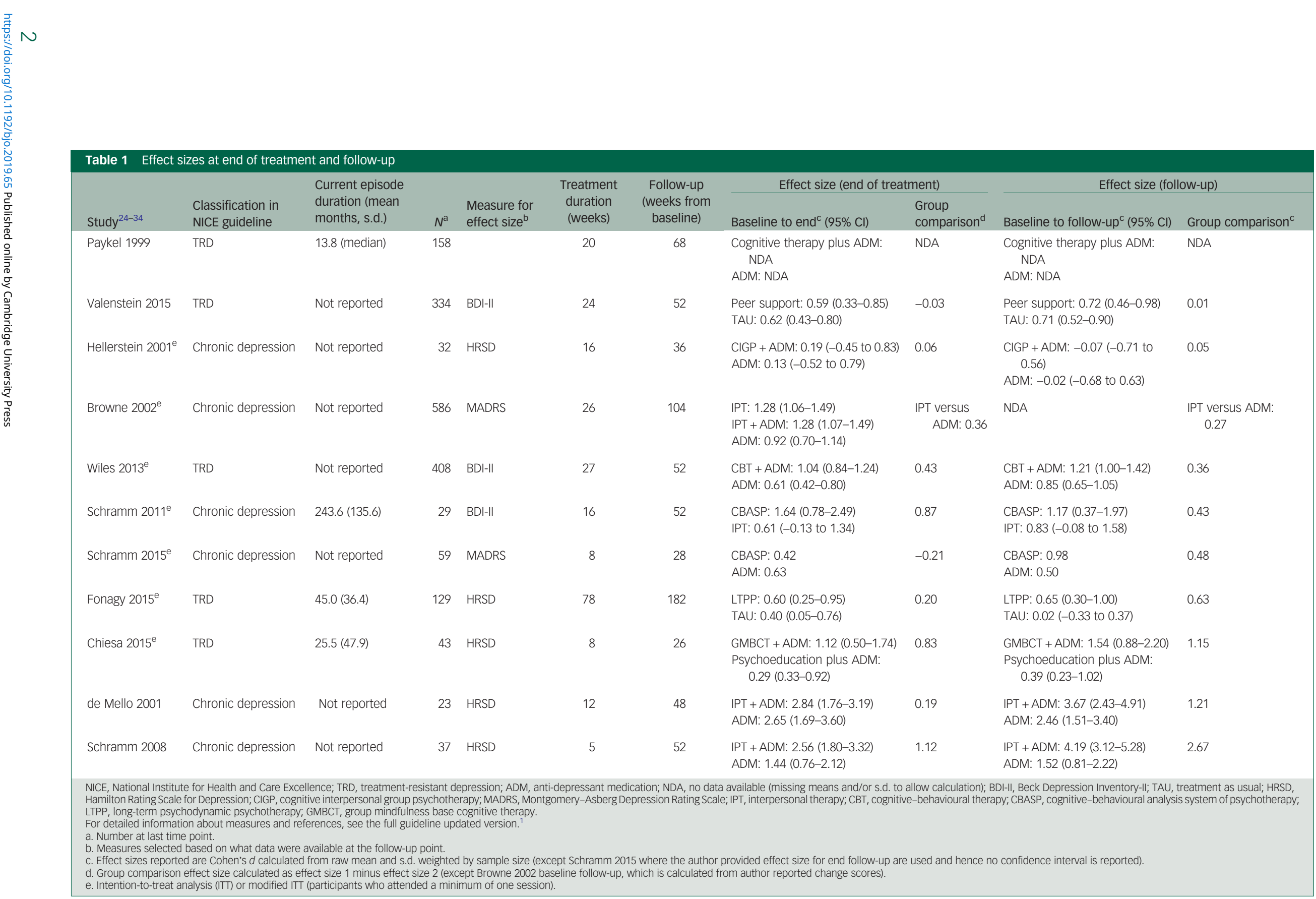

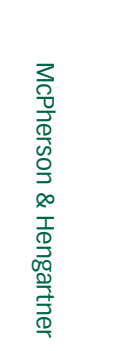


on quality where participants were not blinded; stakeholders argue this systematically prejudices psychological treatment trials in which concealment of the treatment arm is impossible.

Although it remains necessary to be cautious about the tentative findings presented, it is also possible to consider other forms of longitudinal data that appear to support the findings. Discontinuation trials, for example, were designed to test the efficacy of staying on antidepressants long-term compared with stopping and switching to placebo. About a third of patients remaining on antidepressants relapse within 6 months of maintenance therapy. ${ }^{5}$ Remaining on antidepressants for more than 18 months appears to prevent immediate relapse but, when antidepressants are then discontinued, recurrence is very common. Indeed, recurrence is more likely the longer the antidepressant has been taken and is more likely for patients using antidepressants than for trial participants who remitted while on placebo. ${ }^{6,7}$ These findings could be a result of 'oppositional tolerance' or neurobiological adaptation to antidepressants worsening the long-term course of depression. ${ }^{8}$ The picture is further complicated, however, because any trial examining 'relapse' as an outcome of stopping antidepressants could be conflating relapse with withdrawal symptoms. ${ }^{9}$

Various naturalistic cohort studies show that long-term antidepressant use has worse outcomes than short-term antidepressant use or non-pharmacological treatments. ${ }^{10,11}$ Moreover, it appears to be difficult to stop antidepressants after extended use; ${ }^{12}$ some patients using antidepressants may develop dependency ${ }^{13}$ and patients using antidepressants long term report feeling addicted. ${ }^{14}$ Safety reviews find that long-term antidepressant use is associated with serious health risks, including obesity, hepatotoxicity and cardiovascular events. ${ }^{8}$

Meta-analyses of direct comparisons in randomised controlled trials for (recurrent) major depressive disorder (MDD) consistently show that, in protecting against relapse in the long term, psychological treatment is superior to maintenance antidepressant use. ${ }^{15}$ In combination for up to 6 months, antidepressants plus psychological treatment appears to be more effective for MDD than psychological treatment or antidepressants alone; however, long-term follow-ups reveal that beyond 6 months, there is no advantage of combination treatment over psychological treatment alone. ${ }^{16}$ Two reviews also found that (mindfulness-based) cognitive-behavioural therapy following antidepressant discontinuation was more effective than antidepressant continuation. ${ }^{17,18}$

Treatment comparison studies suggest that long-term psychological treatment irrespective of modality generates large effect sizes over the long term for MDD and chronic depression. For example, comparisons of cognitive-behavioural therapy and psychoanalytic psychotherapy find roughly equal but strong effect sizes after 1 year ${ }^{19}$ and 3 years. ${ }^{20}$ Similarly, a systematic review found that long-term relapse rates in depression over 2 years are lower for psychological treatments than other interventions. ${ }^{21}$ In contrast, less than $10 \%$ of patients treated with antidepressants achieve sustained remission over 1 year in representative realworld effectiveness trials. ${ }^{22}$ A systematic review of naturalistic studies of antidepressant use of at least 10 years' duration revealed that only about a quarter of people improved or remitted, and another quarter were classified as severely impaired and illfunctioning. ${ }^{23}$

\section{Conclusion}

If it is possible to agree that good-quality, long-term trial data is the best possible evidence for long-term conditions, then the paucity and variable quality of this data should not be a reason to exclude it in analyses used to inform depression guideline recommendations. Tentative analyses of long-term outcome data reveal an important clinical picture, which is supported by evidence from other forms of longitudinal research. Although the evidence remains to be appropriately evaluated with GRADE methodology, it is nevertheless important to consider it in the guideline because of the effect NICE guidelines have globally on patient care as well as ongoing and future research priorities.

Susan McPherson (1D, PhD, Researcher, School of Health and Social Care, University of Essex, UK; Michael P. Hengartner, PhD, Senior Lecturer and Researcher, School of Applied Psychology, Zurich University of Applied Sciences, Switzerland

Correspondence: Susan McPherson, School of Health and Social Care, University of Essex, Wivenhoe Park, Colchester, CO4 3SQ, UK. Email: smcpher@essex.ac.uk

First received 10 Jun 2019, final revision 13 Aug 2019, accepted 14 Aug 2019

\section{Data availability}

Sources of information used were NICE guideline drafts, documents and appendices all available on the NICE website. ${ }^{1}$ Specifically, Appendix $\mathrm{J} 5$ and $\mathrm{J} 6$ were used, which detail all trials reviewed in the TRD and chronic depression categories. See https://www.nice.org.uk/ guidance/gid-cgwave0725/documents/addendum-appendix-9 and https://www.nice.org.uk/ guidance/gid-cgwave0725/documents/addendum-appendix-10. Note that Town 2017 was included in the 'Full guideline updated' but omitted from Appendix J5.

\section{References}

1 National Institute for Health and Care Excellence (NICE). Depression in adults: treatment and management. Addendum Consultation. NICE, 2018 (Www. nice.org.uk/guidance/gid-cgwave0725/documents).

2 National Institute for Health and Care Excellence (NICE). Depression in adults: treatment and management. Consultation comments and responses - second consultation period. NICE, 2018 (https://www.nice.org.uk/guidance/gidcgwave0725/documents/consultation-comments-and-responses-2).

3 Wise J. NICE guidance on depression: 35 organisations demand 'full and proper revision'. BMJ 2019; 365: 12356

4 McPherson S. A NICE game of Minecraft: philosophical flaws underpinning UK depression guideline methodology. Med Humanit 2019, in press.

5 Gueorguieva R, Chekroud A, Krystal J. Trajectories of relapse in randomised, placebo-controlled trials of treatment discontinuation in major depressive disorder : an individual patient-level data meta-analysis. Lancet Psychiatry 2017; 4: 230-7.

6 Andrews $\mathrm{P}$, Kornstein S, Halberstadt C, Gardener C, Neale M. Blue again: perturbational effects of antidepressants suggest monoaminergic homeostasis in major depression. Front Psychol 2011; 2: 159.

7 El-Mallakh R, Briscoe B. Studies of long-term use of antidepressants: how should the data from them be interpreted? CNS Drugs 2012; 26: 97-109.

8 Fava GA, Offidani E. The mechanisms of tolerance in antidepressant action. Prog Neuro Psychopharmacol Biol Psychiatry 2011; 35: 1593-602.

9 Cohen D, Recalt AM. Discontinuing psychotropic drugs from participants in randomized controlled trials: a systematic review. Psychother Psychosom 2019; 88: 96-104.

10 Hengartner MP, Angst J, Rossler W. Antidepressant use prospectively relates to a poorer long-term outcome of depression: results from a prospective community cohort study over 30 years. Psychother Psychosom 2018; 87: 181-3.

11 Bockting $\mathrm{CLH}$, ten Doesschate $\mathrm{MC}$, Spijker J, Koeter MWJ, Schene $\mathrm{AH}$. Continuation and maintenance use of antidepressants in recurrent depression. Psychother Psychosoms 2008; 77: 17-26.

12 Groot PC, van Os J. Antidepressant tapering strips to help people come off medication more safely. Psychosis 2018; 10: 142-5.

13 Fava GA, Gatti A, Belaise C, Guidi J, Offidani E. Withdrawal symptoms after selective serotonin reuptake inhibitor discontinuation: a systematic review. Psychother Psychosom 2015; 84: 72-81.

14 Read J, Cartwright C, Gibson K. Adverse emotional and interpersonal effects reported by 1829 New Zealanders while taking antidepressants. Psychiatry Res 2014; 216: 67-73.

15 Biesheuvel-Leliefeld KE, Kok GD, Bockting CL, Cuijpers $P$, Hollon SD, van Marwijk HW, et al. Effectiveness of psychological interventions in preventing recurrence of depressive disorder: meta-analysis and meta-regression. J Affect Disord 2015; 174: 400-10. 
16 Karyotaki E, Smit Y, Holdt Henningsen K, Huibers MJ, Robays J, de Beurs D, et al. Combining pharmacotherapy and psychotherapy or monotherapy for majo depression? A meta-analysis on the long-term effects. J Affect Disord 2016 194: $144-52$

17 Guidi J, Tomba E, Fava GA. The sequential integration of pharmacotherapy and psychotherapy in the treatment of major depressive disorder: a meta-analysis of the sequential model and a critical review of the literature. Am J Psychiatry 2016; 173(2): 128-37.

18 Kuyken W, Warren FC, Taylor RS, Whalley B, Crane C, Bondolfi G, et al. Efficacy of mindfulness-based cognitive therapy in prevention of depressive relapse: an individual patient data meta-analysis from randomized trials. JAMA Psychiatry 2016; 73(6): 565-74

19 Driessen E, Van HL, Don FJ, Peen J, Kool S, Westra D, et al. The efficacy of cog nitive-behavioral therapy and psychodynamic therapy in the outpatient treatment of major depression: a randomized clinical trial. Am J Psychiatry 2013; 170: $1041-50$

20 Leuzinger-Bohleber $M$, Hautzinger $M$, Fiedler $G$, Keller $W$, Bahrke $U$, Kallenbach $L$, et al. Outcome of psychoanalytic and cognitive-behavioural long-term therapy with chronically depressed patients: a controlled trial with preferential and randomized allocation. Can J Psychiatry 2019; 64: 47-58.

21 Steinert C, Hofmann M, Kruse J, Leichsenring F. Relapse rates after psychotherapy for depression - stable long-term effects? A meta-analysis. J Affect Disord 2014; 168: 107-18.

22 Pigott HE, Leventhal AM, Alter GS, Boren JJ. Efficacy and effectiveness of antidepressants: current status of research. Psychother Psychosom 2010; 79: $267-79$.

23 Hughes S, Cohen D. A systematic review of long term studies of drug treated and non-drug treated depression. J Affect Disord 2009; 118: 9-18.

24 Paykel ES, Scott J, Teasdale JD, Johnson AL, Garland A, Moore R, et al. Prevention of relapse in residual depression by cognitive therapy: a controlled trial. Arch Gen Psych 1999; 56(9): 829-35.

25 Valenstein M, Pfeiffer PN, Brandfon S, Walters H, Ganoczy D, Kim HM, et al. Augmenting ongoing depression care with a mutual peer support intervention versus self-help materials alone: a randomized trial. Psych Serv 2015; 67(2): 236-9.
26 Hellerstein DJ, Little SA, Samstag LW, Batchelder S. Adding group psychotherapy to medication treatment in dysthymia. J Psychother Pract Res 2001; 10(2): 93.

27 Browne G, Steiner M, Roberts J, Gafni A, Byrne C, Dunn E, et al. Sertraline and/or interpersonal psychotherapy for patients with dysthymic disorder in primary care: 6-month comparison with longitudinal 2-year follow-up of effectiveness and costs. J Aff Dis 2002; 68(2): 317-30.

28 Schramm E, Zobel I, Schoepf $D$, Fangmeier $T$, Schnell $K$, Walter $H$, et al. Cognitive behavioral analysis system of psychotherapy versus escitalopram in chronic major depression. Psychother Psychosom 2015; 84(4): 227-40.

29 Schramm E, Zobel I, Dykierek P, Kech S, Brakemeier EL, Külz A, et al. Cognitive behavioral analysis system of psychotherapy versus interpersonal psychotherapy for early-onset chronic depression: a randomized pilot study. J Aff Dis 2011; 129(1): 109-16.

30 Wiles N, Thomas L, Abel A, Ridgway N, Turner N, Campbell J, et al. Cognitive behavioural therapy as an adjunct to pharmacotherapy for primary care based patients with treatment resistant depression: results of the CoBalT randomised controlled trial. Lancet 2013; 381(9864): 375-84.

31 Fonagy P, Rost F, Carlyle JA, McPherson S, Thomas R, Pasco Fearon RM, et al. Pragmatic randomized controlled trial of long-term psychoanalytic psychotherapy for treatment-resistant depression: the Tavistock Adult Depression Study (TADS). World Psychiatry 2015; 14(3): 312-21.

32 Chiesa A, Castagner V, Andrisano C, Serretti A, Mandelli L, Porcelli S, et al. Mindfulness-based cognitive therapy vs. psycho-education for patients with major depression who did not achieve remission following antidepressant treatment. Psychiatry Res 2015; 226(2): 474-83.

33 de Mello MF, Myczcowisk LM, Menenzes PR. A randomized controlled trial comparing moclobemide and moclobemide plus interpersonal psychotherapy in the treatment of dysthymic disorder. J Psychother Pract Res 2001; 10(2): 117.

34 Schramm E, Schneider D, Zobel I, van Calker D, Dykierek P, Kech S, et al. Efficacy of interpersonal psychotherapy plus pharmacotherapy in chronically depressed inpatients. J Aff Dis 2008; 109(1): 65-73. 